\title{
DIREÇÃO HORÁRIA PREDOMINANTE E DE RAJADA DE VENTO EM LOCALIDADES DOS CAMPOS GERAIS DO PARANÁ E ARREDORES
}

\author{
BURGARDT, Solange - solangeburgardt@yahoo.com.br \\ UEPG - Universidade Estadual de Ponta Grossa \\ LEITE, Maysa de Lima - mleite@uepg.br \\ UEPG - Universidade Estadual de Ponta Grossa \\ VIRGENS FILHO, Jorim Sousa das - jvirgens@uepg.br \\ UEPG - Universidade Estadual de Ponta Grossa
}

\begin{abstract}
Resumo
Para verificar se existe relação entre as direções de vento predominante e rajadas com o período do dia e/ou meses do ano, em sete localidades do Estado do Paraná, foram analisados os dados horários registrados pelas estações meteorológicas respectivas a cada localidade. Os dados foram então agrupados em dois períodos noturnos e dois períodos diurnos. Cada período compreende 6 horas. Dessa forma, verificou-se que Cândido de Abreu, Fernandes Pinheiro e Jaguariaíva apresentaram tendência de variação da direção dos ventos relacionada ao período do dia, especificamente no período da tarde. Em contrapartida, Lapa, Ponta Grossa, Telêmaco Borba e União da Vitória apresentaram variação da direção dos ventos relacionada aos meses do ano, em maior proporção.
\end{abstract}

PALAVRAS-CHAVE: direção predominante, direção de rajada, vento horário.

HOURLY DIRECTION OF THE PREDOMINANT AND GUST WIND IN LOCATIONS OF CAMPOS GERAIS OF PARANÁ AND SURROUNDINGS

\section{Abstract}

To check whether relationship between the directions of prevailing wind and gusts with the time of day and/or months of the year, in seven localities of Paraná State, it was analyzed hourly data recorded by weather stations at each location. Data were then grouped in two night periods and two day periods. Each period correspond to 6 hours. Thus, it was observed that Cândido de Abreu, Fernandes Pinheiro and Jaguariaíva tended to change in wind direction related to the period of the day, specifically in the afternoon. In other wway the places of Lapa, Ponta Grossa, Telêmaco Borba and União da Vitória showed variation of wind direction related to the months of the year, in greater proportion.

Keywords: predominant direction, direction gusts, hourly wind.

\section{INTRODUÇÃO}

Ventos constituem uma das variáveis meteorológicas com influência direta no cotidiano humano e no ambiente como um todo. Desenvolver mais estudos sobre o comportamento dessa variável é o ponto inicial para que seja possível, por exemplo, prever, com base na tendência geral, os eventos extremos que poderão gerar consequências, principalmente à agricultura e engenharia civil (PEREIRA et al., 2010). A partir desses estudos também se percebe que são várias as possíveis aplicabilidades do conhecimento do comportamento eólico de determinada região.

Desde a melhor localização para implantação de indústrias, visando a mínima emissão de gases direcionados ao meio urbano (CONCEIÇÃo, 2008), assim como a localização mais apropriada para aterros sanitários e afins, tendo basicamente o mesmo objetivo, o monitoramento de fumaças e propagação de focos de queimadas (CONCEIÇÃO, 2008; BARBANO et al., 2003) e de fontes poluidoras do ar (LEITE \& VIRGENS FILHO, 2006), previsão da deriva, para os arredores da cultura, onde são aplicados produtos químicos (CONCEIÇÃO, 2008) e a partir disso, analisar quais os locais mais apropriados para a distribuição de diferentes cultivares no campo (GALVANI et al., 1999), até mesmo pelo fato de que além de defensivos, o vento pode carrear pólen, doenças, pragas agrícolas, 
(MUNHOZ \& GARCIA, 2008), e ainda intercambiar gás carbônico, vapor d'água e calor sensível entre a vegetação e a atmosfera, o que influencia no crescimento da vegetação (LIMA \& GALVANI, 2008).

Por outra ótica, ainda pode auxiliar no posicionamento de quebra ventos (CONCEIÇÃO, 2008; LEITE \& VIRGENS FILHO, 2006; GALVANI et al., 1999; MUNHOZ \& GARCIA, 2008), orientação e planejamento do conforto térmico (LEITE \& VIRGENS FILHO, 2006), seja em residências ou em construções diversas (GALVANI et al., 1999).

Os prejuízos e catástrofes que podem resultar principalmente das rajadas, como o acamamento de plantas, erosão eólica dos solos desprovidos de cobertura vegetal (BARBANO, 2003) e inclusive a morte de pequenos animais, como citado por Silva et al. (1997), só podem ser prevenidos por uma estimativa de como, com qual intensidade e quando é mais provável que eventos com tal repercussão e consequências estejam mais tendenciosos a ocorrer.

Diferentes fatores contribuem para a variabilidade da direção dos ventos predominantes e rajadas. Pela Física, é possível citar três deles: gradiente de pressão (que pelo aquecimento desigual do ar, gera diferentes densidades e pressões, fazendo com que o ar se desloque), força de Coriolis (produzida pela movimentação rotacional da Terra) e atrito com a superfície atmosférica (que origina divergências e convergências conforme a altitude da superfície) (GONÇALVES, 2007). Outros fatores como tempo, espaço, situação geográfica, grau de rugosidade da superfície atmosférica, presença ou não de vegetação, clima, estação do ano, período do dia, presença de obstáculos (como por exemplo, construções elevadas) podem exercer influência relevante (VENDRAMINI, 1986; GALVANI et al., 1999). E ainda, nos locais com potencial eólico, dados de direção dos ventos definem 0 melhor posicionamento e dimensionamento das torres de energia, para utilização do vento como fonte de energia (GALVANI et al., 1999).

A direção horária, principalmente para atividades dinâmicas, que dependem da variação que ocorre durante o dia, como por exemplo, o melhor horário para aplicação de defensivos agrícolas são as mais beneficiadas por estudos específicos que evidenciam as várias direções ocorrentes durante o dia. Sabendo-se da importância e da escassez de estudos deste cunho científico, este tem por objetivo traçar um perfil de direção horária do vento predominante e rajadas através da análise das frequências horárias dos ventos para os municípios paranaenses de Cândido de Abreu, Fernandes Pinheiro, Jaguariaíva, Lapa, Ponta Grossa, Telêmaco Borba e União da Vitória.

\section{MATERIAIS E MÉTODOS}

As localidades em estudo estão distribuídas pelo território estadual conforme a Figura 1. Dessas localidades, quatro fazem parte da divisão geográfica, baseada em critérios naturais, dos Campos Gerais do Paraná (Jaguariaíva, Telêmaco Borba, Ponta Grossa e Lapa), e três estão localizadas nos arredores (Cândido de Abreu, Fernandes Pinheiro e União da Vitória) (MELO et al. 2007). 


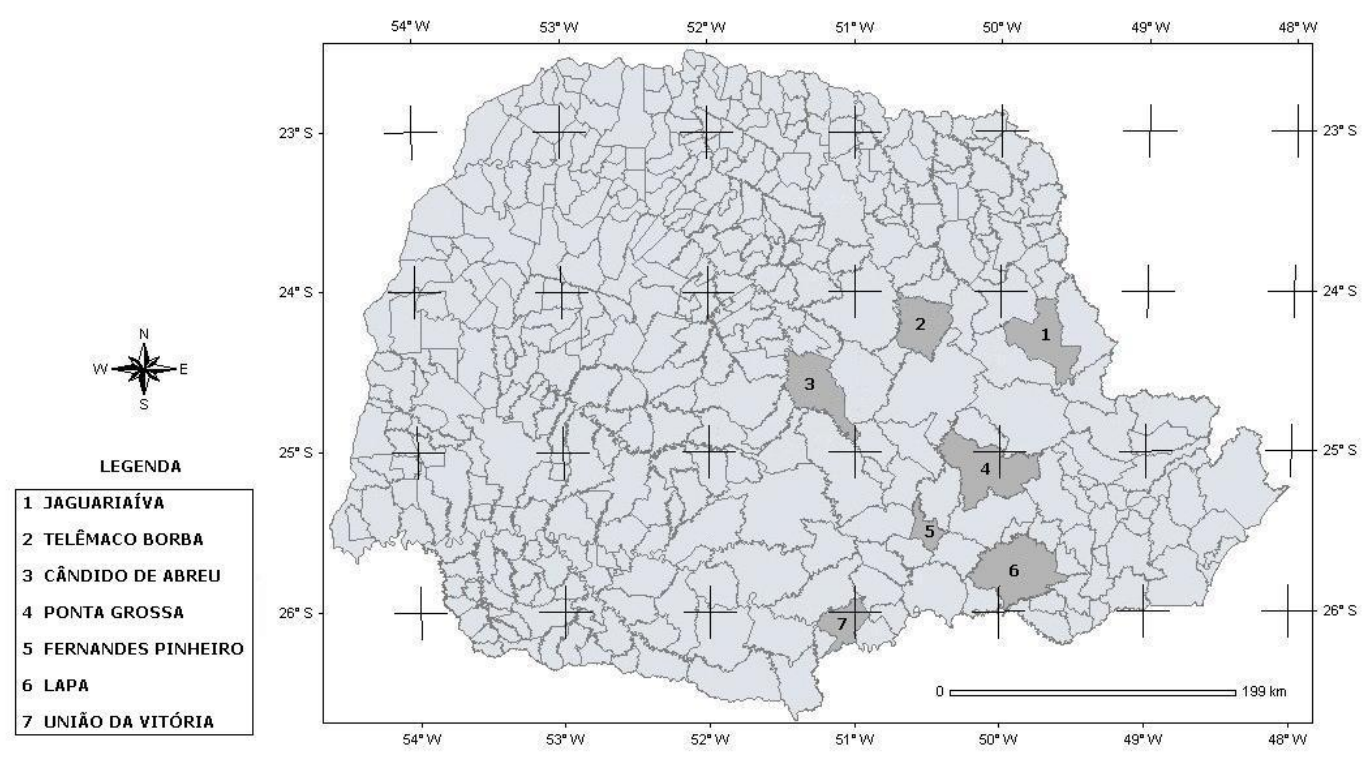

Figura 1 - Localização dos municípios paranaenses estudados.

Os dados utilizados para o desenvolvimento desta pesquisa são provenientes dos registros horários de ventos predominantes e rajadas de sete estações meteorológicas automáticas do Instituto Tecnológico SIMEPAR, correspondentes as localidades estudadas (Figura 1).

Abaixo relacionados (Tabela 1 ), estão as coordenadas geografias e o tempo de registro de cada série de dados.

Tabela 1 - Coordenadas Geográficas das localidades estudadas.

\begin{tabular}{ccccc}
\hline Localidade & Latitude & Longitude & Altitude (metros) & Registro (anos) \\
\hline Cândido de Abreu & $24^{\circ} 63^{\prime} \mathrm{S}$ & $51^{\circ} 25^{\prime} \mathrm{W}$ & 645 & $1997-2007$ \\
Fernandes Pinheiro & $25^{\circ} 45^{\prime} \mathrm{S}$ & $50^{\circ} 58^{\prime} \mathrm{W}$ & 893 & $1998-2007$ \\
Jaguariaíva & $24^{\circ} 23^{\prime} \mathrm{S}$ & $49^{\circ} 68^{\prime} \mathrm{W}$ & 900 & $2000-2007$ \\
Lapa & $25^{\circ} 78^{\prime} \mathrm{S}$ & $49^{\circ} 77^{\prime} \mathrm{W}$ & 910 & $1998-2007$ \\
Ponta Grossa & $25^{\circ} 22^{\prime} \mathrm{S}$ & $50^{\circ} 02^{\prime} \mathrm{W}$ & 886 & $1998-2007$ \\
Telêmaco Borba & $24^{\circ} 33^{\prime} \mathrm{S}$ & $50^{\circ} 62^{\prime} \mathrm{W}$ & 768 & $1997-2007$ \\
União da Vitória & $26^{\circ} 23^{\prime} \mathrm{S}$ & $51^{\circ} 07^{\prime} \mathrm{W}$ & 757 & $1997-2007$ \\
\hline
\end{tabular}

Para descrever probabilisticamente as direções predominantes para a velocidade do vento, foi utilizado o cálculo de frequência relativa simples $\left(\mathrm{fr}_{\mathrm{d}}\right)$ :

$$
\mathrm{fr}_{d}=\frac{\mathrm{f}_{\mathrm{d}}}{\mathrm{n}}
$$

\section{Eq. 1}

onde: " $f_{d}$ " é a frequência absoluta de cada direção e " $n$ " corresponde ao número de observações dentro da análise.

Os dados de vento foram obtidos através de anemômetros instalados a 10 metros de altura, modelo YOUNG 05103-11A, cujos valores obedecem a 
uma sistemática específica de registro. Para tanto, os dados de ventos horários são calculados com base na média dos 10 registros medidos nos últimos 10 segundos de cada hora. E os dados de rajada são obtidos, com o cálculo do maior valor horário das 60 médias dos 10 registros nos 10 últimos segundos, de cada minuto da hora (GONÇALVES, 2007) em que a rajada está referenciada no Banco de Dados (Figura 2).

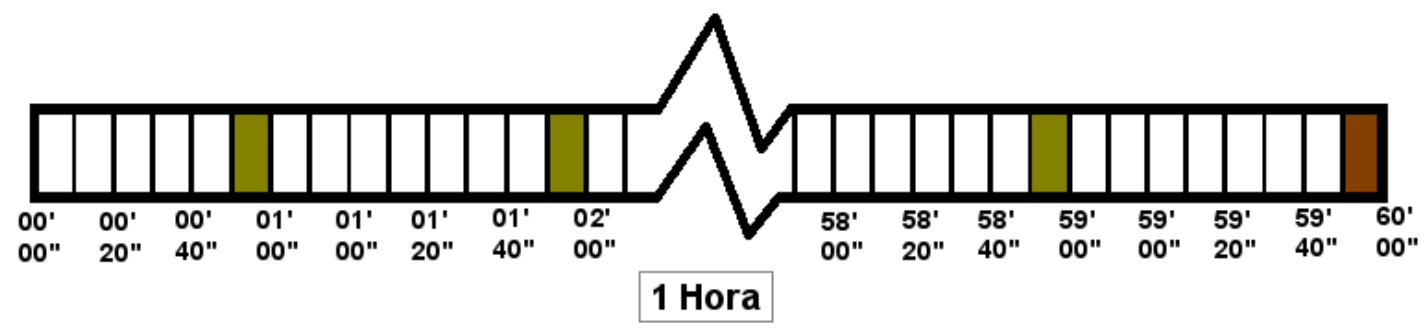

Média dos últimos $10 "$ da hora, utilizada para o cálculo da velocidade do vento

Média dos últimos 10" do minuto, utilizada para o cálculo da velocidade da rajada

Figura 2 - Representação gráfica da amostragem das variáveis no Banco de Dados do SIMEPAR.

Fonte: Gonçalves, 2007.

A partir desses registros, foi analisada cada hora, em cada mês do ano, e os dados das 24 horas foram classificados em períodos designados por período 1 ( $0-5$ horas), 2 ( $6-11$ horas), 3 ( $12-17$ horas), 4 (18-23 horas) para maior detalhamento quanto à possível relação entre a direção predominante e o período do dia, constituindo dois períodos diurnos e dois noturnos. A escolha desta análise por período foi feita com base na interferência da presença ou ausência da radiação solar nos gradientes de pressão atmosférica, no decorrer das 24 horas do dia, e possíveis modificações nas direções de vento predominante e rajada.

Dando seguimento ao estudo, foram construídas tabelas que demonstram objetivamente os resultados obtidos.

\section{RESULTADOS E DISCUSSÃO}

Durante a triagem dos dados horários de vento predominante e rajada nas sete localidades em estudo, observou-se que, apesar da grande variabilidade nas direções durante o dia, sendo ocasionada em grande parte, justamente pela especificidade que este registro proporciona, em alguns casos houve uma tendência de que as mesmas direções apareçam em certos períodos que geralmente abrangem as mesmas horas. Esses períodos foram então individualizados, com base na observação geral das oscilações que ocorreram com as direções no decorrer das 24 horas e também no decorrer do ano.

\section{- Cândido de Abreu:}

Ao observar os resultados obtidos para o município de Cândido de Abreu (Tabela 2), fica confirmado que não houve significativa diferença entre as direções horárias de ventos predominantes e rajadas, nesta localidade. 
Alguns casos apresentam oscilação entre duas direções, isto se deve ao fato de que em algumas ocasiões $50 \%$ dos registros no período apresentam uma direção e os outros $50 \%$ apresentam outra direção. Nesta localidade, porém, são oscilações pequenas, que permanecem ainda no mesmo quadrante de direção, com exceção apenas no mês de julho, no período 3, do registro de rajadas, quando houve oscilação entre as direções sudeste e noroeste. Pode-se perceber que os períodos 1 e 2 apresentam SE como direção mais frequente, e quando analisado o registro de vento predominante, observou-se que no período 3, a direção que aparece em mais de $50 \%$ dos registros no período, é NW. É possível perceber também que nos meses de setembro e outubro, praticamente não houve oscilação entre as direções, dentro dos períodos especificados, e nem entre os registros de vento predominante e rajada. Nos demais meses, ocorre principalmente no período 3 uma direção diferente, que não pode ser explicada por oscilação dentro do quadrante. O período das 12 às 17 horas, geralmente apresenta o maior grau de insolação do dia, o que pode ter ocasionado alterações no gradiente de pressão, pelo aquecimento diferencial em comparação aos outros períodos do dia, e assim gerado movimentação do ar com direção diferente. Outra observação também pode ser feita no período 4 da maior parte dos meses. Ocorre geralmente direção $E$, sendo que nos períodos 1 e 2 é constante a direção SE como mais frequente tanto para vento predominante como rajada. Este período corresponde ao intervalo de horas em que se inicia o período de resfriamento noturno, porém ainda com certa quantidade de energia acumulada no período diurno. Com o passar das horas, a perda de calor armazenado se acentua, permitindo, mais uma vez observar alterações no gradiente de pressão.

No estudo sobre ventos extremos, Gonçalves (2007) relata que Cândido de Abreu apresenta comportamento regionalmente independente. Por ser um município com grande parte da economia baseada na agropecuária, é importante entender a dinâmica dos ventos e principalmente como ocorrem os eventos extremos, para que o planejamento das atividades relacionadas seja realizado com menores chances de imprevistos e prejuízos.

Tabela 2 - Direções do vento predominante e rajada em Cândido de Abreu em quatro períodos horários.

\begin{tabular}{|c|c|c|c|c|c|c|c|c|c|}
\hline & \multicolumn{2}{|c|}{ Dominante } & \multicolumn{2}{|c|}{ Rajada } & & \multicolumn{2}{|c|}{ Dominante } & \multicolumn{2}{|c|}{ Rajada } \\
\hline & período & direção & período & direção & & período & direção & período & direção \\
\hline \multirow{5}{*}{ JAN } & 1 & SE & 1 & SE & & 1 & SE & 1 & SE \\
\hline & 2 & SE & 2 & SE & JUL & 2 & SE & 2 & SE \\
\hline & 3 & W & 3 & W/NW & & 3 & NW & 3 & SE/NW \\
\hline & 4 & SE & 4 & SE & & 4 & $E$ & 4 & E \\
\hline & 1 & $\mathrm{E} / \mathrm{SE}$ & 1 & $\mathrm{E} / \mathrm{SE}$ & & 1 & SE & 1 & SE \\
\hline \multirow[t]{4}{*}{ FEV } & 2 & SE & 2 & SE & AGO & 2 & SE & 2 & SE \\
\hline & 3 & W & 3 & W & & 3 & NW & 3 & NW \\
\hline & 4 & $E$ & 4 & $E$ & & 4 & $E$ & 4 & $E$ \\
\hline & 1 & SE & 1 & SE & & 1 & SE & 1 & SE \\
\hline \multirow[t]{3}{*}{ MAR } & 2 & SE & 2 & SE & SET & 2 & SE & 2 & SE \\
\hline & 3 & NW & 3 & NW & & 3 & SE & 3 & SE \\
\hline & 4 & $E$ & 4 & $E$ & & 4 & $\mathrm{E} / \mathrm{SE}$ & 4 & SE \\
\hline
\end{tabular}




\begin{tabular}{|c|c|c|c|c|c|c|c|c|c|}
\hline \multirow{4}{*}{ ABR } & 1 & $\mathrm{SE}$ & 1 & SE & \multirow{3}{*}{ OUT } & 1 & SE & 1 & SE \\
\hline & 2 & SE & 2 & SE & & 2 & SE & 2 & SE \\
\hline & 3 & NW & 3 & NW & & 3 & SE & 3 & SE \\
\hline & 4 & $E$ & 4 & E & & 4 & SE & 4 & SE \\
\hline \multirow{4}{*}{ MAIO } & 1 & SE & 1 & SE & \multirow{4}{*}{ NOV } & 1 & SE & 1 & $\mathrm{SE}$ \\
\hline & 2 & SE & 2 & SE & & 2 & SE & 2 & SE \\
\hline & 3 & SE & 3 & NW & & 3 & W/NW & 3 & NW \\
\hline & 4 & $\mathrm{E}$ & 4 & E & & 4 & SE & 4 & $\mathrm{E} / \mathrm{SE}$ \\
\hline \multirow{4}{*}{ JUN } & 1 & SE & 1 & $\mathrm{E} / \mathrm{SE}$ & \multirow{4}{*}{ DEZ } & 1 & SE & 1 & SE \\
\hline & 2 & SE & 2 & SE & & 2 & SE & 2 & SE \\
\hline & 3 & NW & 3 & NW & & 3 & NW & 3 & NW \\
\hline & 4 & E & 4 & E & & 4 & E & 4 & E \\
\hline
\end{tabular}

\section{- Fernandes Pinheiro:}

Para a localidade de Fernandes Pinheiro, também foi observada a mesma tendência de que o período 3 apresente direção diferente dos demais períodos (Tabela 3). Na maioria dos meses, a direção observada com maior frequência, nos períodos 1,2 e 4 é SE. Com a ressalva do mês de junho, que apresenta vento predominante oscilando entre três direções no período 4. NW oscilante com SE, aparece nos dois registros horários de ventos, no mesmo período, sendo que no registro de vento predominante a oscilação foi, além dessas com a direção N. Setembro se mantém em SE constantemente em todos os períodos. Outubro tem direção diferente de SE apenas no período 3 da direção horária de vento predominante, onde surge a direção $E$. Mas de modo geral observa-se que a oscilação de direção ocorre majoritariamente no período 3, quando $\mathrm{SE}$, presente na maior parte dos demais períodos, é substituído em grande parte por NW.

Fernandes Pinheiro também é um município cuja base econômica é baseada principalmente na agropecuária. Da mesma forma, essas informações auxiliarão na realização das atividades relacionadas.

Além disso, a FLONA (Floresta Nacional de Irati) está localizada em Fernandes Pinheiro (maior parte) e Teixeira Soares. É uma unidade de conservação que compreende uma área de 3.618,21 ha, com espécies nativas, local onde são desenvolvidas pesquisas científicas principalmente para o uso sustentável dos recursos (TETTO et al., 2010). Por estar inserida em um município onde há realizações de atividades ligadas à agricultura e pecuária, onde possivelmente a queima controlada é uma atividade comum, seja para limpeza dos resíduos de colheita ou renovação de pastagem, conhecer a oscilação da direção dos ventos durante o dia é de grande relevância para que uma atividade ainda considerada cotidiana, não se torne um desastre ecológico, com perda de cobertura vegetal e da biodiversidade existente em Fernandes Pinheiro.

Tabela 3 - Direções do vento predominante e rajada em Fernandes Pinheiro em quatro períodos horários.

\begin{tabular}{|c|c|c|c|c|c|c|c|c|c|}
\hline & \multicolumn{2}{|c|}{ Dominante } & \multicolumn{2}{|c|}{ Rajada } & & \multicolumn{2}{|c|}{ Dominante } & \multicolumn{2}{|c|}{ Rajada } \\
\hline & período & direção & período & direção & & período & direção & período & direção \\
\hline & 1 & SE & 1 & SE & & 1 & $\mathrm{SE}$ & 1 & SE \\
\hline JAN & 2 & SE & 2 & SE & JUL & 2 & SE & 2 & SE \\
\hline & 3 & NW & 3 & NW/SE & & 3 & NW & 3 & NW \\
\hline & 4 & SE & 4 & SE & & 4 & SE & 4 & SE \\
\hline & 1 & SE & 1 & SE & & 1 & SE & 1 & SE \\
\hline
\end{tabular}




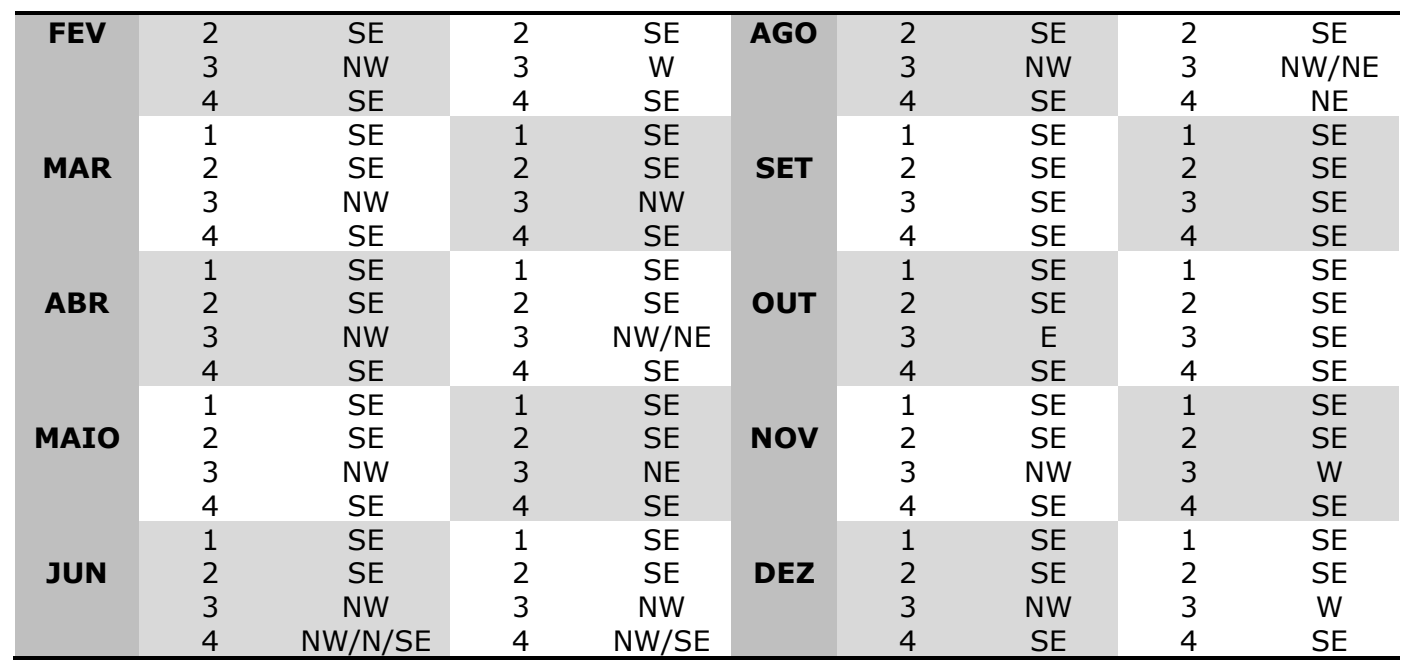

\section{- Jaguariaíva}

Na localidade de Jaguariaíva observa-se a mesma tendência de oscilação de direção no período 3 (Tabela 4). Há também uma maior oscilação de direção nos outros períodos, ocorrendo durante um intervalo de 6 horas, mais de uma direção. Essa oscilação, que na maior parte ocorre tanto para vento dominante quanto rajada, está presente principalmente no período 2, que corresponde das horas 6 a 11, e oscila geralmente entre as direções SW e NE. Apesar de apresentar maior número de oscilações, é possível perceber que a tendência geral é que os períodos 1,2 e 4 tenham a direção SW como a mais frequente, em vento dominante e rajada, e que no período 3 surja a direção NW apareça mais vezes.

Maack (1981) descreve que $38,1 \%$ dos ventos em Jaguariaíva são do quadrante sul e $29,2 \%$ são do quadrante norte, resultados de 43 anos de observação de registros diários. Ainda disponibiliza as porcentagens referentes a cada uma das direções, mesmo com baixos valores as que apresentam maiores porcentagens são SE $(17,4 \%)$ e NW $(17,4 \%)$, pois as demais direções também tem porcentagens relevantes. Desta forma, observamos que a tendência observada por Maack continua sendo confirmada no presente trabalho, com o diferencial de que as direções nos pontos colaterais não foram as mesmas que resultaram desta pesquisa. Tal resultado se deve, muito provavelmente, pela diferença nas escalas dos registros de dados utilizados, onde é possível perceber que detalhes evidenciados na escala horária, se perdem quando se trabalha com médias diárias. No presente trabalho, percebe-se que de forma geral, SW e NE são as direções que aparecem com maior frequência.

Neste município se localiza o PEC (Parque Estadual do Cerrado), com 426,62 ha. localizado na Área de Proteção Ambiental Estadual da Escarpa Devoniana (400.000 ha.), sendo uma unidade de conservação que protege os últimos remanescentes desse bioma no Estado do Paraná (STRAUBE et al., 2005). Sabendo a dinâmica dos ventos ao longo do dia, é possível planejar melhor as ações para combater, por exemplo, o incêndio acidental ou provocado, de forma a não desencadearem maiores danos, grande perca de cobertura vegetal e atingir o meio urbano. 
Tabela 4 - Direções do vento predominante e rajada em Jaguariaíva em quatro períodos horários.

\begin{tabular}{|c|c|c|c|c|c|c|c|c|c|}
\hline & \multicolumn{2}{|c|}{ Dominante } & \multicolumn{2}{|c|}{ Rajada } & & \multicolumn{2}{|c|}{ Dominante } & \multicolumn{2}{|c|}{ Rajada } \\
\hline & período & direção & período & direção & & período & direção & período & direção \\
\hline \multirow{5}{*}{ JAN } & 1 & SW & 1 & SW & & 1 & SW & 1 & SW \\
\hline & 2 & NE & 2 & SW/NE & JUL & 2 & SW & 2 & SW \\
\hline & 3 & $\mathrm{NE}$ & 3 & NE & & 3 & NE & 3 & $\mathrm{NE}$ \\
\hline & 4 & sW & 4 & S & & 4 & SW & 4 & SW \\
\hline & 1 & SW & 1 & SW & & 1 & SW & 1 & SW \\
\hline \multirow[t]{4}{*}{ FEV } & 2 & SW/NE & 2 & SW/NE & AGO & 2 & SW & 2 & SW \\
\hline & 3 & N/NE & 3 & $\mathrm{NE}$ & & 3 & $\mathrm{NE}$ & 3 & $\mathrm{NE}$ \\
\hline & 4 & SW & 4 & SW & & 4 & SW & 4 & SW \\
\hline & 1 & SW & 1 & SW & & 1 & SW & 1 & SW \\
\hline \multirow[t]{4}{*}{ MAR } & 2 & SW/NE & 2 & SW/NE & SET & 2 & SW & 2 & SW \\
\hline & 3 & $\mathrm{NE}$ & 3 & $\mathrm{NE}$ & & 3 & $\mathrm{NE}$ & 3 & $\mathrm{NE}$ \\
\hline & 4 & sW & 4 & SW & & 4 & SW & 4 & SW \\
\hline & 1 & SW & 1 & SW & & 1 & SW & 1 & SW \\
\hline \multirow[t]{4}{*}{ ABR } & 2 & sW & 2 & SW & OUT & 2 & SW/NE & 2 & SW/NE \\
\hline & 3 & $\mathrm{NE}$ & 3 & NE & & 3 & $\mathrm{NE}$ & 3 & $\mathrm{NE}$ \\
\hline & 4 & SW & 4 & SW & & 4 & SW & 4 & SW \\
\hline & 1 & SW & 1 & SW & & 1 & SW & 1 & SW \\
\hline \multirow[t]{4}{*}{ MAIO } & 2 & SW & 2 & SW & NOV & 2 & $\mathrm{NE}$ & 2 & NE \\
\hline & 3 & $\mathrm{NE}$ & 3 & $\mathrm{NE}$ & & 3 & $\mathrm{NE}$ & 3 & NE \\
\hline & 4 & SW & 4 & SW & & 4 & SW & 4 & S/SW \\
\hline & 1 & SW & 1 & SW & & 1 & SW & 1 & SW \\
\hline \multirow[t]{3}{*}{ JUN } & 2 & SW & 2 & SW & DEZ & 2 & NE & 2 & $\mathrm{NE}$ \\
\hline & 3 & NE & 3 & NE & & 3 & NE & 3 & NE \\
\hline & 4 & sW & 4 & SW & & 4 & SW & 4 & S/SW \\
\hline
\end{tabular}

\section{- Lapa}

O município da Lapa foge um pouco da tendência apresentada pelas outras localidades supracitadas (Tabela 5). Setembro, outubro, novembro, dezembro e janeiro apresentam constantemente a direção $\mathrm{E}$ em todos os períodos, exceto o período 2 do registro horário da direção de rajada no mês de dezembro, quando oscila $\mathrm{E}$ e NE. Os dois registros horários de ventos nos meses de fevereiro, março, maio e junho, e o registro horário de rajada no mês de julho apresentam a direção NW no período 3. Mesmo assim essa observação não tem o mesmo significado encontrado na análise das localidades acima discutidas, pois houve maior diferença de direções entre os demais períodos. Poderia ter a mesma tendência em alguns desses meses, mas como também surgem direções diferentes nos outros períodos, em função dos meses, como se observa entre março e junho, por exemplo, fica difícil definir se existe realmente alguma tendência no período 3. É possível observar que mesmo que $E$ seja a direção mais frequente no geral, há meses em que a direção NE aparece com maior frequência em grande parte dos períodos, como ocorre nos meses de maio, junho, julho e agosto. Pereira et al. (2010), em seu estudo utilizando dados de direção diária do vento, diz que E é a direção predominante para Lapa. Beruski et al. (2009), também utilizando dados diários de 19 anos de registro da direção dos ventos em Lapa, apresenta uma tabela com as freqüências de cada direção no decorrer dos meses, enfatizando que no período de outubro a abril a direção para vento predominante com maior frequência é $E$, mas no período de maio a setembro a direção com maior 
frequência é NE. Talvez pelo fato de que a série de dados naquele estudo tenha sido maior, ou porque a especificidade das séries diárias é menor em comparação às séries horárias, houve algumas divergências entre os resultados de Beruski et al. (2009) e os resultados aqui obtidos. Os meses de setembro a abril apresentaram direção $E$ com mais frequência, independente do período do dia, da mesma forma que os meses de maio a agosto apresentaram a direção NE. Nas direções de rajada, Beruski et al. (2009) obtiveram maior oscilação, mas com E e NE mantendo-se como as direções com maiores freqüências em função dos meses.

Tabela 5 - Direções do vento predominante e rajada em Lapa em quatro períodos horários.

\begin{tabular}{|c|c|c|c|c|c|c|c|c|c|}
\hline & \multicolumn{2}{|c|}{ Dominante } & \multicolumn{2}{|c|}{ Rajada } & & \multicolumn{2}{|c|}{ Dominante } & \multicolumn{2}{|c|}{ Rajada } \\
\hline & período & direção & período & direção & & período & direção & período & direção \\
\hline & 1 & E & 1 & E & & 1 & $\mathrm{NE}$ & 1 & $\mathrm{NE}$ \\
\hline \multirow[t]{4}{*}{ JAN } & 2 & $\mathrm{E}$ & 2 & $E$ & JUL & 2 & $\mathrm{NE}$ & 2 & $\mathrm{NE}$ \\
\hline & 3 & $E$ & 3 & E & & 3 & $\mathrm{NE}$ & 3 & NW \\
\hline & 4 & $\mathrm{E}$ & 4 & $\mathrm{E}$ & & 4 & $\mathrm{E}$ & 4 & $E$ \\
\hline & 1 & $\mathrm{E}$ & 1 & $\mathrm{E}$ & & 1 & $\mathrm{NE}$ & 1 & $\mathrm{NE}$ \\
\hline \multirow[t]{4}{*}{ FEV } & 2 & $E$ & 2 & $\mathrm{E} / \mathrm{NE}$ & AGO & 2 & $\mathrm{NE}$ & 2 & $\mathrm{NE}$ \\
\hline & 3 & NW & 3 & NW & & 3 & $\mathrm{~N}$ & 3 & $\mathrm{NE}$ \\
\hline & 4 & $\mathrm{E}$ & 4 & E & & 4 & $\mathrm{E}$ & 4 & $\mathrm{E}$ \\
\hline & 1 & E & 1 & E & & 1 & E & 1 & $E$ \\
\hline \multirow[t]{4}{*}{ MAR } & 2 & $E$ & 2 & $E$ & SET & 2 & $\mathrm{E}$ & 2 & $\mathrm{E}$ \\
\hline & 3 & NW & 3 & NW & & 3 & $\mathrm{E}$ & 3 & $\mathrm{E}$ \\
\hline & 4 & $\mathrm{E}$ & 4 & E & & 4 & E & 4 & $E$ \\
\hline & 1 & $E$ & 1 & $E$ & & 1 & $\mathrm{E}$ & 1 & $\mathrm{E}$ \\
\hline \multirow[t]{4}{*}{ ABR } & 2 & $N E$ & 2 & $\mathrm{NE}$ & OUT & 2 & E & 2 & E \\
\hline & 3 & E & 3 & E & & 3 & E & 3 & $E$ \\
\hline & 4 & $\mathrm{E}$ & 4 & $E$ & & 4 & E & 4 & $\mathrm{E}$ \\
\hline & 1 & $\mathrm{NE}$ & 1 & $\mathrm{NE}$ & & 1 & $\mathrm{E}$ & 1 & $\mathrm{E}$ \\
\hline \multirow[t]{4}{*}{ MAIO } & 2 & $\mathrm{E} / \mathrm{NE}$ & 2 & $\mathrm{NE}$ & NOV & 2 & E & 2 & $E$ \\
\hline & 3 & NW & 3 & NW & & 3 & $\mathrm{E}$ & 3 & $\mathrm{E}$ \\
\hline & 4 & $\mathrm{E}$ & 4 & $\mathrm{E}$ & & 4 & $\mathrm{E}$ & 4 & $\bar{E}$ \\
\hline & 1 & NE & 1 & NE & & 1 & $\mathrm{E}$ & 1 & $\mathrm{E}$ \\
\hline \multirow{3}{*}{ JUN } & 2 & $\mathrm{NE}$ & 2 & $\mathrm{NE}$ & DEZ & 2 & E & 2 & $\mathrm{E} / \mathrm{NE}$ \\
\hline & 3 & NW & 3 & NW & & 3 & $\mathrm{E}$ & 3 & $E$ \\
\hline & 4 & $E$ & 4 & E & & 4 & $\mathrm{E}$ & 4 & $\mathrm{E}$ \\
\hline
\end{tabular}

Esses resultados podem ser de grande valia para o município, tendo em vista ser o quarto maior em área territorial do Estado, ter sua base econômica ligada às atividades agrícolas (sementes, grãos e cereais), com a maior área plantada de frutas de caroço do Paraná, e ainda atividades ligadas à pecuária leiteira (LEITE et al., 2010). Assim sendo, a disposição de cultivares no campo

e das árvores nos pomares e a aplicação de defensivos são exemplos de atividades que poderão ser mais bem planejadas e executadas com o conhecimento da direção horária dos ventos nos diferentes períodos do dia.

\section{- Ponta Grossa}

Para a cidade de Ponta Grossa, também não é possível definir uma tendência com direção diferente apenas em um dos períodos (Tabela 6). Os meses de janeiro e setembro, e o registro horário de rajadas do mês de 
maio apresentam direção discordante apenas no período 3. É possível perceber mais facilmente que os meses de junho, julho e agosto, apresentam, sem oscilação, a direção NE em todos os períodos, e o registro horário de vento predominante do mês de outubro apresenta constância na direção SE. Wagner, et al. (1989), utilizando dados de médias mensais, sazonais e anuais nos períodos de 1973/76 - 1982/85/86 obtiveram a direção NE nos resultados de todas as formas de análise. Leite \& Virgens Filho (2006), em seu estudo utilizando dados de 13 anos de registro diário da localidade de Ponta Grossa, concluíram que NE é a direção mais frequente ao longo do ano seguida pelas ocorrências decrescentes de NW e E. Pereira et al. (2010), utilizando registros de dados de médias diárias, apresentam também a direção NE como predominante para Ponta Grossa, não observando tendência de oscilação significativa entre os meses ou estações do ano. No presente trabalho o que foi observado é que, a maioria dos meses apresenta a mesma direção no período noturno, havendo maior oscilação e surgimento de outras direções no período diurno. Os meses de janeiro, fevereiro, março, abril, setembro e dezembro tiveram como mais frequente, nos

Tabela 6 - Direções do vento predominante e rajada em Ponta Grossa em quatro períodos horários.

\begin{tabular}{|c|c|c|c|c|c|c|c|c|c|}
\hline & \multicolumn{2}{|c|}{ Dominante } & \multicolumn{2}{|c|}{ Rajada } & & \multicolumn{2}{|c|}{ Dominante } & \multicolumn{2}{|c|}{ Rajada } \\
\hline & período & direção & período & direção & & período & direção & período & direção \\
\hline \multirow{4}{*}{ JAN } & 1 & SE & 1 & SE & \multirow{4}{*}{ JUL } & 1 & NE & 1 & NE \\
\hline & 2 & SE & 2 & SE & & 2 & NE & 2 & NE \\
\hline & 3 & NW & 3 & NW & & 3 & NE & 3 & NE \\
\hline & 4 & SE & 4 & SE & & 4 & NE & 4 & NE \\
\hline & 1 & SE & 1 & SE & \multirow{4}{*}{ AGO } & 1 & NE & 1 & NE \\
\hline \multirow[t]{4}{*}{ FEV } & 2 & NE & 2 & NE/SE & & 2 & NE & 2 & NE \\
\hline & 3 & $\mathrm{~N}$ & 3 & NW & & 3 & NE & 3 & NE \\
\hline & 4 & SE & 4 & SE & & 4 & NE & 4 & NE \\
\hline & 1 & SE & 1 & SE & \multirow{3}{*}{ SET } & 1 & SE & 1 & SE \\
\hline \multirow[t]{4}{*}{ MAR } & 2 & NE & 2 & NE & & 2 & SE & 2 & SE \\
\hline & 3 & N/SE & 3 & $\mathrm{~N}$ & & 3 & NE & 3 & NE \\
\hline & 4 & SE & 4 & SE & & 4 & SE & 4 & SE \\
\hline & 1 & SE & 1 & SE & \multirow{4}{*}{ OUT } & 1 & SE & 1 & SE \\
\hline \multirow[t]{4}{*}{ ABR } & 2 & SE & 2 & SE & & 2 & SE & 2 & SE \\
\hline & 3 & NE/SE & 3 & NE & & 3 & SE & 3 & NE \\
\hline & 4 & NE/SE & 4 & SE & & 4 & SE & 4 & SE \\
\hline & 1 & NE & 1 & SE & \multirow{3}{*}{ NOV } & 1 & SE & 1 & SE \\
\hline \multirow[t]{4}{*}{ MAIO } & 2 & SE & 2 & SE & & 2 & NE & 2 & NE/SE \\
\hline & 3 & NW & 3 & NE & & 3 & SE & 3 & SE \\
\hline & 4 & NE & 4 & NE & & 4 & SE & 4 & SE \\
\hline & 1 & NE & 1 & NE & \multirow{4}{*}{ DEZ } & 1 & SE & 1 & SE \\
\hline \multirow[t]{3}{*}{ JUN } & 2 & NE & 2 & NE & & 2 & NE & 2 & NE \\
\hline & 3 & NE & 3 & NE & & 3 & N/NW & 3 & NE/SE \\
\hline & 4 & NE & 4 & NE & & 4 & SE & 4 & SE \\
\hline
\end{tabular}

dois registros horários dos ventos, a direção SE nos períodos 1 e 4, que correspondem as horas 18 a 5, ou seja, o período noturno. O mês de maio teve a direção NE no registro horário de vento predominante, aparecendo com a maior freqüência no período noturno.

Ponta Grossa, com produção de milho e soja em grande escala (LEITE et al., 2010) tem uma parcela relevante da economia baseada nessas 
atividades, e como já foi citado, principalmente as atividades agrícolas são beneficiadas por informações mais específicas do comportamento eólico, seja para determinar o melhor período do dia para aplicação de defensivos agrícolas ou mesmo para dispor de forma acertada as melhores posições para cada cultivar dentro da área plantada, tendo em vista, principalmente a polinização cruzada (MUNHOZ \& GARCIA, 2008).

O Parque Estadual de Vila Velha (PEVV) e Cachoeira do Buraco do Padre são alguns dos atrativos naturais presentes na região. O PEVV é uma área com esculturas naturais de arenito, mas apesar de sugestões de alguns autores, não há evidências da erosão eólica na formação das mesmas (MELO et al., 2002). Porém, por serem áreas verdes abertas à visitação e também próximas de áreas de cultivo agrícola, é relativamente frequente a ocorrência de incêndios principalmente no PEVV (ANTONELLI, 2011). Na Cachoeira do Buraco do Padre as ocorrências de incêndios são menores. Desta forma, conhecendo a direção do vento e oscilações, o controle de eventos como este é facilitado.

\section{- Telêmaco Borba}

Na localidade de Telêmaco Borba, não é possível observar nenhuma grande tendência quanto à direção dos ventos em relação ao período do dia (Tabela 7). Alguns casos isolados ocorreram como no registro horário de vento predominante nos meses de fevereiro e março, e nos registros horários de rajada nos meses de setembro, janeiro e abril, quando o período 3 tem direção mais discordante que os demais. Apenas os meses de outubro, novembro e dezembro pareceram manter essa possível tendência, tendo nos períodos 1, 2 e 4 a mesma direção (SE) e no período 3 revelando direção diferenciada, sem oscilação para $\mathrm{N}$ em outubro e NW em novembro e dezembro. Wagner et al., (1989), em seu estudo desenvolvido na mesma localidade no período de 1972/78-1982/86, utilizando dados de direção diária, obteve como direção predominante SE, pelas médias mensais, sazonais e anuais. O que se pode acrescentar a partir do presente trabalho, é que apesar de SE ser a direção com maior freqüência, é possível perceber uma grande oscilação ocorrente durante o dia, mesmo que os resultados revelem apenas a oscilação em menos de $50 \%$ no período de 6 horas.

Tabela 7 - Direções do vento predominante e rajada em Telêmaco Borba em quatro períodos horários.

\begin{tabular}{|c|c|c|c|c|c|c|c|c|c|}
\hline & \multicolumn{2}{|c|}{ Dominante } & \multicolumn{2}{|c|}{ Rajada } & & \multicolumn{2}{|c|}{ Dominante } & \multicolumn{2}{|c|}{ Rajada } \\
\hline & período & direção & período & direção & & período & direção & período & direção \\
\hline \multirow{5}{*}{ JAN } & 1 & $E / S E$ & 1 & SE & & 1 & S/SE & 1 & S/SE \\
\hline & 2 & $\mathrm{E} / \mathrm{SE} / \mathrm{N}$ & 2 & SE & JUL & 2 & SE & 2 & SE \\
\hline & 3 & NW & 3 & NW & & 3 & $\mathrm{~N}$ & 3 & $\mathrm{~N}$ \\
\hline & 4 & SE & 4 & SE & & 4 & S & 4 & SW \\
\hline & 1 & SE & 1 & SE & & 1 & $\mathrm{~S}$ & 1 & $\mathrm{~S}$ \\
\hline \multirow[t]{4}{*}{ FEV } & 2 & SE & 2 & SE & AGO & 2 & SE & 2 & SE \\
\hline & 3 & N/NW & 3 & NW & & 3 & $\mathrm{~N}$ & 3 & $\mathrm{~N}$ \\
\hline & 4 & SE & 4 & S/SE & & 4 & $\mathrm{~S}$ & 4 & SW \\
\hline & 1 & SE & 1 & SE & & 1 & SE & 1 & SE \\
\hline MAR & 2 & SE & 2 & SE & SET & 2 & SE & 2 & SE \\
\hline
\end{tabular}




\begin{tabular}{|c|c|c|c|c|c|c|c|c|c|}
\hline \multirow{6}{*}{ ABR } & 3 & $\mathrm{~N}$ & 3 & NW & \multirow{5}{*}{ OUT } & 3 & $\mathrm{~N}$ & 3 & $\bar{N}$ \\
\hline & 4 & SE & 4 & $S / S E$ & & 4 & SE & 4 & $\mathrm{~S}$ \\
\hline & 1 & SE & 1 & SE & & 1 & SE & 1 & SE \\
\hline & 2 & SE & 2 & SE & & 2 & SE & 2 & SE \\
\hline & 3 & $\mathrm{~N}$ & 3 & NW & & 3 & $\mathrm{~N}$ & 3 & $\mathrm{~N}$ \\
\hline & 4 & $\mathrm{~S}$ & 4 & SE & & 4 & SE & 4 & SE \\
\hline \multirow{3}{*}{ MAIO } & 1 & $\mathrm{~S}$ & 1 & SE & \multirow{4}{*}{ NOV } & 1 & SE & 1 & SE \\
\hline & 2 & SE & 2 & SE & & 2 & SE & 2 & SE \\
\hline & 3 & $\mathrm{~N}$ & 3 & $\mathrm{~N}$ & & 3 & NW & 3 & NW \\
\hline \multirow{5}{*}{ JUN } & 4 & $\mathrm{~S}$ & 4 & S/SW & & 4 & SE & 4 & SE \\
\hline & 1 & SE & 1 & SE & \multirow{4}{*}{ DEZ } & 1 & SE & 1 & SE \\
\hline & 2 & SE & 2 & $E$ & & 2 & $\mathrm{SE}$ & 2 & SE \\
\hline & 3 & $\mathrm{~N}$ & 3 & $\mathrm{~N}$ & & 3 & NW & 3 & NW \\
\hline & 4 & $\mathrm{~S}$ & 4 & SW & & 4 & SE & 4 & $\mathrm{SE}$ \\
\hline
\end{tabular}

Telêmaco Borba é o segundo município quanto a área de florestas nativas e de reflorestamento do Estado, primeiro na produção de toras de madeira e está entre as cidades que concentram a maioria das indústrias do setor madeireiro regional (OLIVEIRA, 2008), além de ter a indústria Klabin de papel e celulose instalada dentro da área abrangente por esse município. Desta forma, as informações referentes a direção horária dos ventos para tal localidade são relevantes quanto se realiza as atividades de reflorestamento, por exemplo, na disposição dentro da área destinada a essa função, e também para o planejamento urbanístico, para que o avanço da cidade se dê longe das instalações industriais e dos locais para onde o vento leva os gases tóxicos.

\section{- União da Vitória}

No município de União da Vitória, foi observado que a direção SE aparece com maior constância em relação aos períodos do dia, não havendo muitos casos de oscilação dentro das 6 horas, e poucas direções diferentes ocorrentes entre os períodos no decorrer do dia (Tabela 8). O período 3 com direção diferente em relação aos demais períodos, ocorreu apenas nos meses de fevereiro (direção de rajada), março (nos dois registros de direção, mas com

Tabela 8 - Direções do vento predominante e rajada em União da Vitória em quatro períodos horários.

\begin{tabular}{|c|c|c|c|c|c|c|c|c|c|}
\hline & \multicolumn{2}{|c|}{ Dominante } & \multicolumn{2}{|c|}{ Rajada } & & \multicolumn{2}{|c|}{ Dominante } & \multicolumn{2}{|c|}{ Rajada } \\
\hline & período & direção & período & direção & & período & direção & período & direção \\
\hline & 1 & $\mathrm{SE}$ & 1 & $\mathrm{SE}$ & & 1 & $\mathrm{SE}$ & 1 & $\mathrm{NE}$ \\
\hline \multirow[t]{4}{*}{ JAN } & 2 & SE & 2 & SE & JUL & 2 & SE & 2 & SE \\
\hline & 3 & SE & 3 & SE & & 3 & SE & 3 & SE \\
\hline & 4 & SE & 4 & $E / S E$ & & 4 & $\mathrm{SE}$ & 4 & $\mathrm{E}$ \\
\hline & 1 & SE & 1 & SE & & 1 & E/SE & 1 & $\mathrm{E} / \mathrm{NE}$ \\
\hline \multirow[t]{4}{*}{ FEV } & 2 & SE & 2 & SE & AGO & 2 & SE & 2 & $\mathrm{E}$ \\
\hline & 3 & SE & 3 & NW & & 3 & SE & 3 & SE \\
\hline & 4 & SE & 4 & SE & & 4 & $\mathrm{E}$ & 4 & $\mathrm{E}$ \\
\hline & 1 & SE & 1 & SE & & 1 & SE & 1 & SE \\
\hline \multirow{4}{*}{ MAR } & 2 & SE & 2 & SE & SET & 2 & SE & 2 & SE \\
\hline & 3 & SE/NW & 3 & NW & & 3 & E/SE & 3 & SE \\
\hline & 4 & SE & 4 & $\mathrm{E}$ & & 4 & SE & 4 & $\mathrm{E} / \mathrm{SE}$ \\
\hline & 1 & SE & 1 & SE & & 1 & SE & 1 & SE \\
\hline ABR & 2 & SE & 2 & SE & OUT & 2 & SE & 2 & $\mathrm{E} / \mathrm{SE}$ \\
\hline
\end{tabular}




\begin{tabular}{cccccccccc}
\hline & 3 & $\mathrm{SE}$ & 3 & $\mathrm{SE}$ & & 3 & $\mathrm{SE}$ & 3 & $\mathrm{SE}$ \\
& 4 & $\mathrm{SE}$ & 4 & $\mathrm{E}$ & & 4 & $\mathrm{SE}$ & 4 & $\mathrm{E} / \mathrm{SE}$ \\
\multirow{4}{*}{ MAIO } & 1 & $\mathrm{SE}$ & 1 & $\mathrm{SE}$ & \multirow{2}{*}{$\mathrm{S}$} & 1 & $\mathrm{SE}$ & 1 & $\mathrm{SE}$ \\
& 2 & $\mathrm{SE}$ & 2 & $\mathrm{SE}$ & NOV & 2 & $\mathrm{SE}$ & 2 & $\mathrm{SE}$ \\
& 3 & $\mathrm{SE}$ & 3 & $\mathrm{SE}$ & & 3 & $\mathrm{E} / \mathrm{SE}$ & 3 & $\mathrm{SE}$ \\
& 4 & $\mathrm{E}$ & 4 & $\mathrm{NE}$ & & 4 & $\mathrm{SE}$ & 4 & $\mathrm{E}$ \\
\multirow{4}{*}{ JUN } & 1 & $\mathrm{SE}$ & 1 & $\mathrm{NE} / \mathrm{SE}$ & & 1 & $\mathrm{SE}$ & 1 & $\mathrm{SE}$ \\
& 2 & $\mathrm{SE}$ & 2 & $\mathrm{SE}$ & $\mathrm{DEZ}$ & 2 & $\mathrm{SE}$ & 2 & $\mathrm{SE}$ \\
& 3 & $\mathrm{SE}$ & 3 & $\mathrm{SE}$ & & 3 & $\mathrm{SE} / \mathrm{NW}$ & 3 & $\mathrm{NW}$ \\
& 4 & $\mathrm{E}$ & 4 & $\mathrm{NE}$ & & 4 & $\mathrm{SE}$ & 4 & $\mathrm{SE}$ \\
\hline
\end{tabular}

oscilação quando vento predominante) e dezembro (nos dois registros de direção, mas com oscilação quando vento predominante). Meses com os 4 períodos apresentando a mesma direção ocorreram em direção horária de vento predominante em janeiro, fevereiro, abril, julho e outubro. Isto mostra que a direção horária de rajada teve maior oscilação que a direção horária de vento predominante, pois não houve nenhuma ocorrência com os 4 períodos apresentando a mesma direção quando analisados os registros de rajadas.

Indústrias seguidas pelas atividades agropecuárias são as atividades que movimentam a economia de União da Vitória (IBGE), da mesma forma como para as demais localidades do estudo com o mesmo perfil, as informações resultantes deste estudo auxiliam no planejamento das atividades no campo (disposição de cultivares, aplicação de defensivos) e no meio urbano (planejamento do crescimento da cidade).

\section{CONCLUSÃO}

As localidades de Cândido de Abreu, Fernandes Pinheiro e Jaguariaíva, apresentam, tanto para rajada quanto para vento predominante horário, a tendência de que o período 3 seja o único com direção diferente dos outros períodos do dia, e estes compartilham a mesma direção entre si. Nas duas primeiras localidades, a direção mais ocorrente é SE, e no período 3 a direção muda para NW. A cidade de Jaguariaíva tem como direção mais frequente SW, e no período 3 a direção NE.

As localidades de Lapa, Ponta Grossa, Telêmaco Borba e União da Vitória não apresentaram esta tendência de diferenciação da direção para vento dominante ou rajada. Houve maior diferença das direções em função dos meses. A localidade de Lapa, com direção $\mathrm{E}$ sendo a mais frequente, teve NE como a direção mais frequente nos meses de maio a agosto. $O$ município de Ponta Grossa teve NE como direção mais frequente, porém de janeiro a abril SE foi a direção mais frequente. A localidade de Telêmaco Borba, com SE sendo a direção mais frequente, apresentou mais oscilações entre os registros de vento predominante e os registros de rajada do que as localidades já mencionadas. O município de União da Vitória, com direção SE sendo a predominante, teve poucas oscilações entre os períodos ou entre os meses. As maiores oscilações percebidas são entre os registros horários de vento predominante e de rajada.

De um modo mais amplo, comparando-se resultados de direções diárias de outros estudos com os resultados horários aqui alcançados, percebe-se que, a determinação de direções diárias para as diversas localidades, prática que acaba sendo mais comum, acaba por revelar uma única direção 
predominante ou de rajada no período de 24 horas, deixando de revelar interferências importantes em algumas localidades, conforme o período do dia, capazes de minimizar prejuízos ou melhorar condutas práticas à partir deste conhecimento.

Com o predomínio de estações automáticas em substituição às antigas estações convencionais, e à facilidade de obtenção de dados horários, espera-se que outros estudos neste sentido possam ser conduzidos, permitindo uma conclusão mais abrangente sobre esta prática.

\section{AGRADECIMENTOS}

Ao Instituto Tecnológico SIMEPAR, por ceder os dados necessários para a realização deste trabalho.

\section{REFERÊNCIAS}

.ANTONELLI, D. Incêndio destrói 60 hectares de Vila Velha. Jornal da manhã, Ponta Grossa, 15 de julho de 2011.

.BARBANO, M. T.; BRUNINI, O.; PINTO, H. S. Direção predominante do vento para a localidade de Campinas - SP. Revista Brasileira de Agrometeorologia, Santa Maria, v.11, n.1, p.123-128, mar. 2003

.CONCEIÇÃO, M. A. F. Condições predominantes dos ventos em Jales (SP). Bento Gonçalves, 2008. 10 p. (Embrapa Uva e Vinho, Comunicado Técnico, 88).

.GALVANI, E.; KLOSOWSKI, E. S.; CUNHA, A. R.; MARTINS, D. Caracterização da direção predominante do vento em Maringá-PR. Revista Brasileira de Agrometeorologia, Santa Maria, v.7, n.1, p. 21-33, 1999.

.GONÇALVES, R. C. 2007. Análise de freqüência regional de ventos extremos no Paraná. Dissertação (Mestrado) - Universidade Federal do Paraná, Setor de Tecnologia, Programa de Pós-Graduação em Engenharia de Recursos Hídricos e Ambiental. Curitiba.

.LEITE, M. L.; ADACHESKI, P. A.; VIRGENS FILHO, J. S. Estudo da variabilidade da precipitação pluvial em municípios da região sul dos Campos Gerais, Paraná, Brasil. Revista Brasileira de Climatologia, v.6, n.6, p.135-149, 2010.

.LEITE, M. L.; VIRGENS FILHO, J. S. Avaliação da velocidade média e direção predominante do vento em Ponta Grossa - PR. Revista Brasileira de Agrometeorologia, v.14, n.2, p. 157-167, 2006.

.LIMA, N. G. B.; GALVANI, E. Variação da direção e velocidade do vento em função da presença de vegetação: os manguezais como estudo de caso. In SIMPÓSIO DE CLIMATOLOGIA GEOGRÁFICA, 8., 2008, Alto Caparaó. Anais..., 2008. p. 573-580.

.MAACK, R. Geografia Física do Estado do Paraná. 2. ed. Rio de Janeiro: Livraria José Olympio Editora, 1981, 450 p.

.MELO, M. S.; BOSETTI, E. P.; GODOY, L. C. PILATTI, F. Vila Velha, PR - Impressionante relevo ruiniforme. In: Schobbenhaus, C.; Campos, D.A.; Queiroz,E.T.; Winge,M.; BerbertBorn,M.L.C. (Edits.). Sítios Geológicos e Paleontológicos do Brasil. 1. ed. Brasília: DNPM/CPRM - Comissão Brasileira de Sítios Geológicos e Paleobiológicos (SIGEP), 2002, v.01. p. 269-277.

.MELO, M. S.; MORO, R. S.; GUIMARÃES, G. B. Patrimônio Natural dos Campos Gerais do Paraná . In: 2007. p. 17-21.

Os Campos Gerais do Paraná. 1. ed. Ponta Grossa: Editora UEPG,

.MUNHOZ, F. C.; GARCIA, A. Caracterização da velocidade e direção predominante dos ventos para a localidade de Ituverava-SP. Revista Brasileira de Meteorologia, v.23, n.1, p. 31-34, mar. 2008.

.OLIVEIRA, M. A. O setor de base florestal paranaense e seus segmentos regionais especializados: 2000/2004. Revista Paranaense de Desenvolvimento, n.115, p.47-78, jul./dez. 2008.

.PEREIRA, L. M. P.; ELY, D. F.; CARAMORI, P. H.; COSTA, A. B. F.; RICCE, W. S. Estudo preliminar da ocorrência de episódio de ventos fortes no Paraná. In: SIMPÓSIO BRASILEIRO DE CLIMATOLOGIA GEOGRÁFICA, 9., 2010, Fortaleza: Anais..., ABClima, 2010.

.SILVA, J. B.; ZANUSSO, J. T.; SILVEIRA, D. L. M.; SCHONS, R. L.; LARROZA, E. G. Estudo da direção e velocidade dos ventos em Pelotas, RS. Revista Brasileira de Agrometeorologia, Santa Maria, v.5, n.2, p.227-235, ago. 1997. 
.STRAUBE, F. C.; URBEN-FILHO, A.; GATTO, C. A avifauna do Parque Estadual do Cerrado (Jaguariaíva, Paraná) e a conservação do cerrado em seu limite meridional de ocorrência. Atualidades Ornitológicas. Ivaiporã, v.127, p.29, 2005.

.TETTO, A. F.; BATISTA, A. C.; NUNES, J. R. S.; SOARES, R. V. Subsídios à prevenção e combate a incêndios florestais com base no comportamento da precipitação pluviométrica na Floresta Nacional de Irati, Paraná. Ciência Florestal, Santa Maria, v.20, n.1, p.33-43, jan.mar. 2010.

.VENDRAMINI, E. Z. 1986. Distribuições probabilísticas de velocidade do vento para avaliação do potencial energético eólico. Tese (Doutorado) - Universidade Estadual Paulista. São Paulo.

.WAGNER, C. S.; BERNARDES, L. R. M.; CORREA, A. R.; BORROZINO, E. Velocidade e direção predominante dos ventos no Estado do Paraná. Londrina, 1989. 56 p. (IAPAR, Boletim Técnico 26). 